THU-94/15

hep-th/9410038

\title{
GAUGE THEORY AND RENORMALIZATION ${ }^{\dagger}$
}

\author{
Gerard 't Hooft \\ Institute for Theoretical Physics \\ University of Utrecht, P.O.Box 80006 \\ 3508 TA Utrecht, the Netherlands
}

\begin{abstract}
Early developments leading to renormalizable non-Abelian gauge theories for the weak, electromagnetic and strong interactions, are discussed from a personal viewpoint. They drastically improved our view of the role of field theory, symmetry and topology, as well as other branches of mathematics, in the world of elementary particles.

\section{Foreword}

Like most other presentations by scientists in this Conference, my account of the most important developments that led towards our present view of the fundamental interactions among elementary particles, is a very personal one, recounting discoveries the author was just about to make when someone else beat him to it. But there is also something else I wish to emphasize. This is the dominant position reoccupied during the last 25 years by Theory, in its relation to Experiment. In particular Renormalized Quantum Field Theory not only fully regained respectability, but has become absolutely essential for understanding those basic facts now commonly known as "The Standard Model". I will limit myself only to the nicest goodies among the many interesting developments in the theory of renormalization, and of those I'll only pick the ones that were of direct importance to me.

The account given here partly overlaps with a similar exposé given two years ago [1] at SLAC.
\end{abstract}

\footnotetext{
$\dagger$ Presented at the International Conference on:

"The History of Original Ideas and Basic Discoveries in Particle Physics"

Erice, Italy, 29 July - 4 August 1994.
} 


\section{Renormalization of QED.}

The early days of renormalization theory are, somewhat disrespectfully, regarded by my generation of physicists as "prehistory". People struggled with the phenomenon of the frequent appearance of "infinities" in their description of relativistic quantumelectromagnetic interactions. It was natural to attempt to reformulate the theories such that these nasty infinities disappeared. A milestone was reached when Hans Bethe [2] found a reasonable looking expression for the Lamb shift in 1947. Julian Schwinger [3] found out how to calculate the first quantum mechanical corrections sytematically. Sin-Itiro Tomonaga [4] and Richard Feynman [5] added a lot to the insights in how renormalization works.

In those days the only quantum field theory known to be manifestly physically important was quantum electrodynamics (QED), describing the interactions between electrons and photons, and a consequence of this was that many features specific to quantum electrodynamics were thought to be essential for renormalizability. A technical difficulty was the problem of "overlapping divergences", the fact that at higher orders infinities of different kinds would get entangled. Freeman Dyson [6] first treated this preoblem in 1949, after which John Ward, Abdus Salam and Steven Weinberg made improvements. Robert Mills and Chen Ning Yang in 1966 discovered that these treatments could still develop flaws at very high order, and they showed how this problem could be handled (ironically, their paper occurs in overlapping issues of Suppl. Progr. Theor. Physics [7]).

An important key in reformulating theories while avoiding infinities would later turn out to be the so-called 'dispersion relations', that had been worked out and discussed by Hans Kramers [8], R. Kronig [9] and Nicolaas van Kampen [10].

But many physicists became quite unhappy with this course of events. The resulting framework very much looked as if it could be summarized as follows:

Start with the "naive", unrenormalized theory. You will see that it contains "infinities". Renormalization simply amounts to "subtracting", or "removing" the infinite terms.

This sounded like: "you hit upon difficulties; just ignore them, cover them up!". As by miracle, the resulting prescriptions are now claimed to be completely unique and selfconsistent. But of course the explanations as to why they work are then lacking, and many textbooks that contain only this version of the argument have added to the wide-spread mistrust and contempt for such an obviously shaky procedure, in spite of its experimental success, which, according to some, had to be accidental [see the extensive review by Cao and Schweber, ref. 11].

With our present understanding we can resolve most of the conceptual difficulties 
people had with renormalization. The complete class of "renormalizable quantum field theories" is now known, and their unified treatment gives much more insight. First of all, all these renormalizable theories should be looked upon as models, which have a built-in limitation in that they can often only be treated as a perturbative series in the coupling constants (with the exception of a very small subclass, the "asymptotically free theories", which, unlike QED, can probably be treated non-perturbatively). The presently adopted "Standard Model" is just a member of this class describing experimental observations extremely well, but it is known not to be infinitely precise and it will need improvements or even a substitution at extremely high energies, see further Section 6. Secondly, the problem of overlapping divergences has now been completely resolved by deriving dispersion relations directly for the Feynman diagrams, a method not so well known but extremely important in the consistency proof of these theories [12].

\section{The Yang-Mills field.}

The successes of renormalization theory for QED were so great that generalizations of this scheme were sought. I presume it was understood that scalar fields could be added, but they were not observed in QED. There do exist strongly interacting scalar (or pseudoscalar) particles, but strong interactions were still very mysterious. The most striking feature of QED is gauge-invariance, and this was what led Yang an Mills to study a theory with a more advanced type of gauge-invariance, the non-Abelian gauge theory ${ }^{\dagger}$. They wrote what I regard as an absolutely beautiful paper [14].

Gauge-invariance is generalized the following way. Assume that there exist more than one type of fermionic fields $\psi(x, t)$ (take the simplest case of just two), which we can arrange as isovectors:

$$
\psi=\left(\begin{array}{c}
\psi_{1} \\
\psi_{2}
\end{array}\right)
$$

Consider transformations of the type

$$
\psi \rightarrow \Omega(\mathbf{x}, t) \psi
$$

where $\Omega$ is a $2 \times 2$ (or possibly larger) matrix. One can then construct the covariant derivative $D_{\mu} \psi$ as follows:

$$
D_{\mu} \psi=\left(\partial_{\mu}-i g B_{\mu}^{a} T^{a}\right) \psi
$$

which transforms just as (2.2) if the new fields $B_{\mu}^{a}$ transform in a very special way. Here $g$ is just some coupling constant, and the matrices $T^{a}$ are the generators of infinitesimal

$\dagger$ The idea was preceded 16 years earlier by Oskar Klein, who derived very similar field equations from a Kaluza-Klein construction [13]. I thank Lev Okun for pointing this out to me. 
rotations $(2.2)$. One can formulate dynamical equations of motion for the new fields $B_{\mu}^{a}$ by first defining the corresponding generalization of the electromagnetic fields $F_{\mu \nu}$. Yang and Mills had first tried

$$
F_{\mu \nu}^{a} \stackrel{?}{=} \partial_{\mu} B_{\nu}^{a}-\partial_{\nu} B_{\mu}^{a} \ldots
$$

but they quickly ran into problems. The equations one would get were not gauge-invariant. To their delight however they found that if one adds to (2.4)

$$
+g f^{a b c} B_{\mu}^{b} B_{\nu}^{c}
$$

where $f^{a b c}$ are the structure constants of the Lie group of matrices $\Omega$ in (2.2), gauge invariance is completely restored.

The field equations are generated by the Lagrangian

$$
\mathcal{L}^{\mathrm{inv}}=-\frac{1}{4} F_{\mu \nu}^{a} F_{\mu \nu}^{a}-\bar{\psi}\left(\gamma_{\mu} D_{\mu}+m\right) \psi
$$

which is indeed invariant under local gauge transformations, and as such a direct generalization of QED.

Since the rigid, space-time independent analog of the transformation group (2.2) (henceforth called the global group) was known as isospin invariance for the strong interactions, Yang and Mills viewed their theory as a scheme to turn isospin into a local symmetry, but they immediately recognized that then there was a problem: the Lagrangian (2.6) describes a massless vector particle with three (or more) components, in general electrically charged as well as neutral ones. In spite of its beauty, this theory was therefore considered to be unrealistic. Besides, since these massless particles interact with each other the theory showed horrible infrared divergencies.

When Yang gave a seminar about his recent result in Princeton, one of the attendants was Wolfgang Pauli. It turned out that Pauli had had some thoughts about such an approach. Yang recollects [15], as soon as he had equation (2.3) on the blackboard,

'Pauli asked: "What is the mass of this field $B_{\mu}$ ?". I said we did not know. Then I resumed my presentation, but soon Pauli asked the same question again. I said something to the effect that that was a very complicated problem, we had worked on it and had come to no definite conclusions. I still remember his repartee: "That is not a sufficient excuse." I was so taken aback that I decided [...] to sit down. There was general embarassment. Finally Oppenheimer said, "We should let Frank proceed". I then resumed, and Pauli did not ask any more questions.'

Clearly, Pauli had been studying very similar schemes himself, but had rejected them because of the mass problem. Proposals to cure this "disease" were made several times. 
Feynman, who looked upon this model as a toy model for quantum gravity, proposed to simply add a small mass term just to avoid the infrared problem [16]:

$$
\mathcal{L}=\mathcal{L}^{\text {inv }}-\frac{1}{2} M^{2}\left(B_{\mu}^{a}\right)^{2} .
$$

Sheldon L. Glashow [17] and Martinus J.G. Veltman [18] proposed to use the same Lagrangian (2.7) as a model for the weak intermediate vector boson. It was hoped that the mass term would not spoil the apparent renormalizability of the Lagrangian (2.5). Probably the philosophy here was that the mass term is only a mild symmetry breaking correction of a kind we see more often in Nature: isospin invariance itself is also softly broken.

Indeed Veltman [17] initially reported progress here: the theory (2.7) is renormalizable at the one-loop level. He made use of field transformations that look like gauge transformations even though the mass term in (2.6) is not gauge invariant:

$$
B_{\mu}^{a \prime}=B_{\mu}^{a}+g f^{a b c} \Lambda^{b} B^{c} \mu-\partial_{\mu} \Lambda^{a} \quad ; \quad \psi^{\prime}=\psi+g \Lambda^{a} T^{a} \psi,
$$

Here $\Lambda$ may be any function of some arbitrarily chosen field variable. The transformation needed to obtain identities among amplitudes for different Feynman diagrams (see Fig. 1) were called Bell-Treiman transformations by Veltman ${ }^{\dagger}$. It would have more appropriate if the identities obtained were called Veltman-Ward identities.

To me it came as a surprise that Veltman managed to renormalize his theory up to one loop with this method. The mass term namely renders the longitudinal part of the gauge field observable, in spite of the fact that the Lagrangian carries no kinetic term for it. This theory should self-destruct. This it does, as Veltman indeed confirmed, but only if you try to renormalize diagrams with two or more loops. To render the "massive Yang-Mills theory" renormalizable, a better theory was needed.

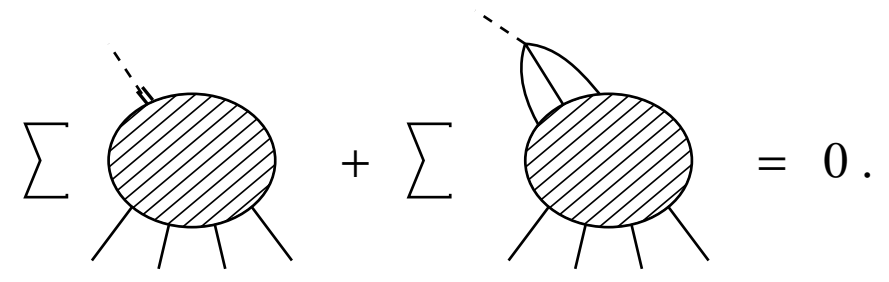

\section{The Gell-Mann Lévy sigma model.}

$\dagger$ using his unique sense of humor. There never existed any references to either Bell or Treiman. 
It was one of those caprices of fate that brought me, as a young student of Veltman's, to the 1970 Cargèse Summer Institute [19]. The champions of renormalization were gathered there to discuss the Gell-Mann Lévy sigma model. This model had been proposed by Murray Gell-Mann and Maurice Lévy [20] in 1960. In order to explain the existence of a partially conserved axial vector current they added a fourth component to the three pion fields, the sigma field, transforming together as a $2 \times 2$ representation of chiral $\mathrm{SU}(2) \times \mathrm{SU}(2)$. The Lagrangian was

$$
\begin{aligned}
& \mathcal{L}(\vec{\pi}, \sigma, \psi, \bar{\psi})=-\frac{1}{2}\left[\partial_{\mu} \vec{\pi}^{2}+\partial_{\mu} \sigma^{2}\right]-\frac{1}{2} \mu_{0}^{2}\left[\vec{\pi}^{2}+\sigma^{2}\right]-\frac{1}{4} \lambda_{0}^{2}\left[\vec{\pi}^{2}+\sigma^{2}\right]^{2} \\
& \quad-\bar{\psi}\left[\gamma_{\mu} \partial_{\mu}+g_{0}\left(\sigma+i \gamma_{5} \vec{\pi} \cdot \vec{\tau}\right)\right] \psi+c \sigma
\end{aligned}
$$

If we take $\mu_{0}^{2}$ here negative then the potential for the scalar fields has the by now familiar dumb-bell shape. The sigma field gets a vacuum expectation value,

$$
\langle\sigma\rangle=F=\left|\mu_{0}\right| / \lambda_{0}
$$

so in a perturbative expansion we write $\sigma=F+s$, and expand in $s$. The nucleon fields $\psi$ get a mass $g_{0} F$, the pions have a tiny mass-square proportional to the small constant $c$, whereas the sigma field $s$ becomes a heavy resonance.

Benjamin W. Lee [21], Jean-Loup Gervais [22] and Kurt Symanzik [23] explained in their Cargèse lectures how this model could be renormalized, and that its beautiful features would not be seriously affected by renormalization. It was clear to me at that time that one can produce mass terms for Yang-Mills fields in a way very similar to this sigma model. I did not ask many questions in this School, but I did ask one question to Benjamin Lee and to Kurt Symanzik: "Do your methods also apply to the Yang-Mills case?" They both gave me the same answer: "If you are Veltman's student, you should ask him; I am not an expert in Yang-Mills theory."

\section{Massless Yang-Mills.}

This I did, as soon as I was back in Utrecht. Now Veltman was skeptical about spontaneous symmetry breakdown in particle theory. His opinion was that if that happens the vacuum would have a tremendously large energy density, and this would give the physical vacuum an enormously large cosmological coupling constant.

But we know it happens in the sigma model, which describes strong interactions pretty nicely. And if it is not symmetry breaking then at least all other vacuum fluctuation effects also contribute to the cosmological coupling constant, not as much as in a weak interaction theory with Higgs mechanism, but still far more than the experimental upper 
bound. The cosmological coupling constant problem should be postponed until we solve quantum gravity; we should not let it affect our theories at the $\mathrm{GeV}$ or $\mathrm{TeV}$ scale.

Veltman still had some reservations concerning theories with spontaneous symmetry breakdown coupled to Yang-Mills fields. He told me he had now nearly convinced himself that you can't add scalar particles to renormalize massive Yang-Mills fields unless you gave them the wrong metric, which would be unacceptable. Would the Higgs theories be the recipe to avoid such dangers? Clearly, I needed to understand these systems better myself. It was then decided what my research program would be. First I would try to really understand all details of the massless, unbroken Yang-Mills system, for which Veltman gave me his blessing, and then I would add the mass, by a "spontaneous local symmetry breaking mechanism".

The status of pure Yang-Mills theory was somewhat vague. Strong formal arguments existed that this "theory"* had to be renormalizable. But there were competing and conflicting theories as to what its Feynman rules were. One paper on this subject was a short Physics Letters paper by Ludwig D. Faddeev and Victor N. Popov [24]. It was all I needed to understand what was going on. Faddeev and Popov argued that a gauge invariant functional integral expression for the amplitudes had to have the form

$$
\Gamma=\int e^{i \int \mathcal{L}^{\mathrm{inv}}(A) \mathrm{d}^{4} x} \prod_{x} \mathrm{~d}^{\ell} A(x),
$$

where $A(x)$ stands for the $\ell$ field components of the gauge and matter system. However, since the integrand is invariant under gauge transformations, one only needs to integrate over the inequivalent field configurations, each being constrained by some gauge condition. As a gauge condition one typically takes

$$
\partial_{\mu} B_{\mu}^{a}=0
$$

If we impose this constraint on the integrand one needs a Jacobian factor however. So if we keep track of the measure, this turns the integral into

$$
\Gamma=C \int e^{i \int \mathcal{L}^{\mathrm{inv}}(A) \mathrm{d}^{4} \mathrm{x}} \prod_{x} \mathrm{~d}^{\ell} A(x) \delta\left(\partial_{\mu} B_{\mu}^{a}(x)\right) \operatorname{det}\left(\frac{\partial\left(\partial_{\mu} B_{\mu}^{a}\right)}{\partial \Lambda}\right)
$$

The theory produces a transverse propagator:

$$
\frac{\delta_{\mu \nu}-\frac{k_{\mu} k_{\nu}}{k^{2}-i \varepsilon}}{k^{2}-i \varepsilon}
$$

* Unfortunately in modern scientific papers the meanings of the words "model" and "theory" are being interchanged for reasons with which I do not agree but that can be explained psychologically. 
Other theories [25] led to a Feynman gauge propagator,

$$
\frac{\delta_{\mu \nu}}{k^{2}-i \varepsilon}
$$

and how this could be related to a functional integral was not clear. More important, I thought, was that none of the existing papers provided for a precise prescription as to how the infinities should be subtracted. The formal arguments were there, but how does it work in practice?

This became the subject of my first publication $[26]^{\ddagger}$. The answer to this question was indeed far from trivial. Several things had to be done. First, the formalism to obtain the Feynman rules from the functional integrals could be simplified. The existing procedure to deduce the ghost Feynman rules from the determinant was not satisfactory. I observed that one can write

$$
(\operatorname{det} \mathcal{M})^{-N}=C \int \mathcal{D} \vec{\phi} \mathcal{D} \vec{\phi}^{*} e^{-\vec{\phi}^{*} \mathcal{M} \vec{\phi}}
$$

where $\phi$ is a complex Lorentz-scalar field with $N$ components $(\mathcal{D} \phi$ stands for the functional measure $\left.\prod_{x} \mathrm{~d} \phi\right)$. One now reads off directly the Feynman rules for closed loops of $\phi$ fields. A factor $N$ goes with each closed loop. Since we want $N$ to be -1 our closed loops will usually go with a factor -1 , just like the rules for fermions. Indeed, one can also write

$$
\operatorname{det} \mathcal{M}=C \int \mathcal{D} \eta \mathcal{D} \bar{\eta} e^{\bar{\eta} \mathcal{M} \eta}
$$

where $\eta$ is an anticommuting (Grassmann) variable.

Next, I could also see how Faddeev and Popov's trick could produce the Feynman gauge. Just take auxiliary field variables $F^{a}$ and impose the gauge

$$
\partial_{\mu} B_{\mu}^{a}=F^{a}
$$

One then sees that

$$
e^{\frac{1}{2}\left(\partial_{\mu} B_{\mu}^{a}\right)^{2}}=\int \mathcal{D} F e^{-\frac{1}{2} F^{2}} \delta\left(\partial_{\mu} B_{\mu}^{a}-F^{a}\right)
$$

To see that the renormalization counter terms do not spoil gauge invariance we needed Ward identities. It turned out to be sufficient to prove identities of the form of Fig. 2.

Since between the accolades reducible and irreducible diagrams must all be added together, these identities are sufficient to restrict all counter terms completely up to gauge

$\ddagger$ In this, and several of my subsequent publications, I benefitted enormously from Veltman’s intensive ineterest and advices. 

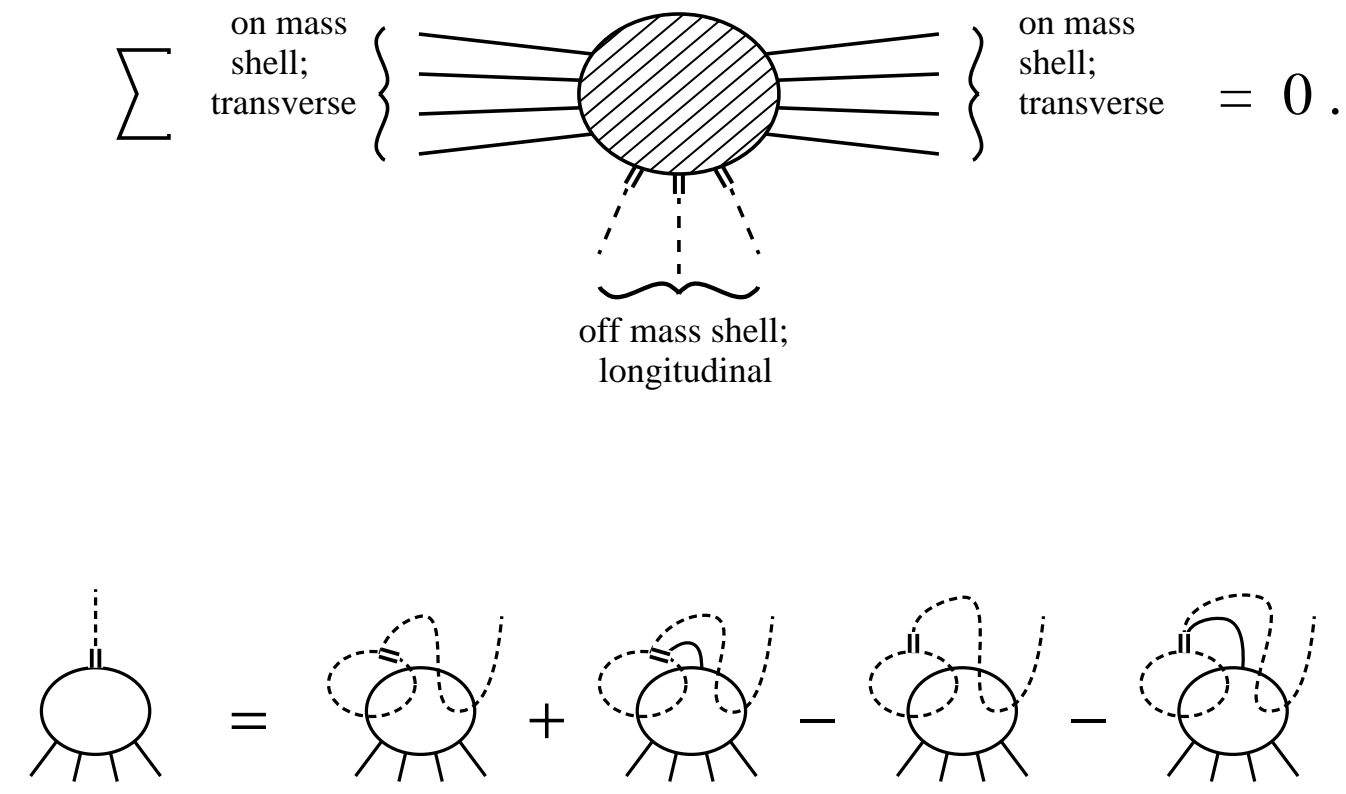

invariant ones. This point was often not realized by later investigators. It becomes obvious if we expand the diagrams of Fig. 2 in terms of one-particle-irreducible ones.

The proof of these Ward identities was much more complicated than the VeltmanWard identities mentioned before, because we had to disentangle carefully the contributions of various ghost lines. See Fig. 3, which was an intermediate step.

I was annoyed that I couldn't use a simple symmetry argument for the proof as Veltman had done for his case. Only much later it was discovered how to do this. C. Becchi, A. Rouet and Raymond Stora found that the underlying symmetry for this identity is an anticommuting one. Their marvelous discovery was this [27]. Take as an invariant Lagrangian for instance

$$
\mathcal{L}^{\text {inv }}=-\frac{1}{4} F_{\mu \nu}^{a} F_{\mu \nu}^{a}-D_{\mu} \phi^{*} D_{\mu} \phi-V\left(\phi, \phi^{*}\right)-\bar{\psi}(\gamma D+m) \psi+\ldots,
$$

and add as a gauge fixing term

$$
\mathcal{L}^{\text {gauge }}=-\frac{1}{2}\left(\ell^{a}\right)^{2},
$$

where $\ell^{a}$ is anything like $\partial_{\mu} B_{\mu}^{a}, B_{4}^{a}$, etc. Introduce the ghost fields $\eta$ and $\bar{\eta}$ which must be anticommuting. Consider then the anticommuting variation:

$$
\begin{aligned}
\delta B_{\mu}^{a} & =\bar{\varepsilon} D_{\mu} \eta^{a} ; \\
\delta \phi & =-i g \bar{\varepsilon} T^{a} \eta^{a} \phi ;
\end{aligned}
$$




$$
\begin{aligned}
\delta \eta^{a} & =\frac{1}{2} g \bar{\varepsilon} f^{a b c} \eta^{b} \eta^{c} \\
\delta \bar{\eta}^{a} & =\bar{\varepsilon} \ell^{a}(B, \phi, \ldots)
\end{aligned}
$$

Here the first lines, eqs. (4.12a) and (4.12b), are just gauge transformations; $\bar{\varepsilon}$ is an infinitesimal, anticommuting symmetry generator. Then the total Lagrangian of the theory when taken to be

$$
\mathcal{L}=\mathcal{L}^{\text {inv }}+\mathcal{L}^{\text {gauge }}+\mathcal{L}^{\text {ghost }}
$$

with

$$
\mathcal{L}^{\text {ghost }}=-\bar{\eta}^{a} \delta \ell^{a}(B, \phi, \ldots \eta)
$$

is invariant under this global transformation. The above identities are nothing but an expression of this invariance, now called BRS invariance.

I had to convince myself that the rules obtained produced a unitary theory. The new identities were sufficient to guarantee this. Just one problem remained: the identities overdetermined the renormalization counter terms. Would there never be a conflict? There was a well-known example of just such a conflict in the literature: the Adler-Bell-Jackiw anomaly. Steve Adler [28], and independently from him John Bell and Roman Jackiw [29], had discovered that diagrams of the kind depicted in Fig. 4 cannot be renormalized in such a way that both the vector current and the axial vector current are conserved. If something like this would happen in a gauge theory there would be deep trouble. I could prove that if no gauge fields are coupled to the axial charge, clashes of this sort will not destroy renormalizability in diagrams with up to one loop. The trick was to use a fifth dimension for the internal lines inside the loop.
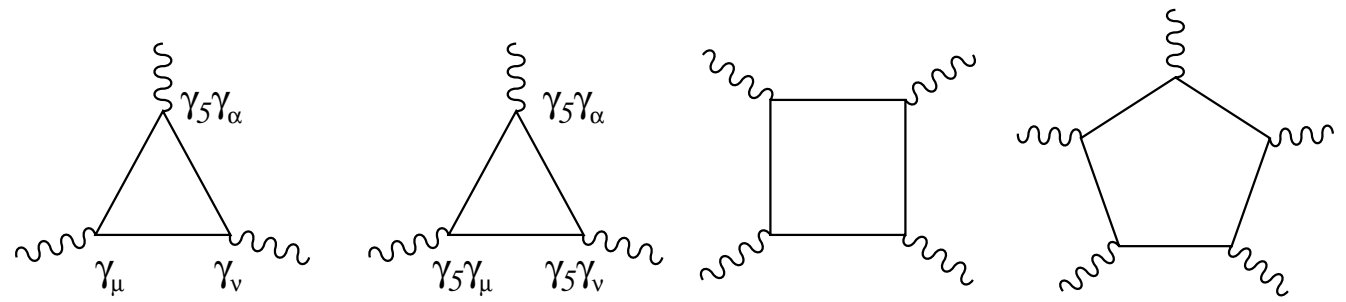

What if you have more than one loop? I tried to use six, seven or more dimensions but this does not work. I was confident that the problem could be solved, but was unable to do it then.

Soon after my paper had come out, two other papers appeared, one by Andrei A. Slavnov [30] and one by John C. Taylor [31] . Both observed that the identities I had 
written down could be generalized. If some of the external lines are neither longitudinal nor on mass shell one gets extra contributions where the ghost line ends up at one of these lines. See Fig. 5.

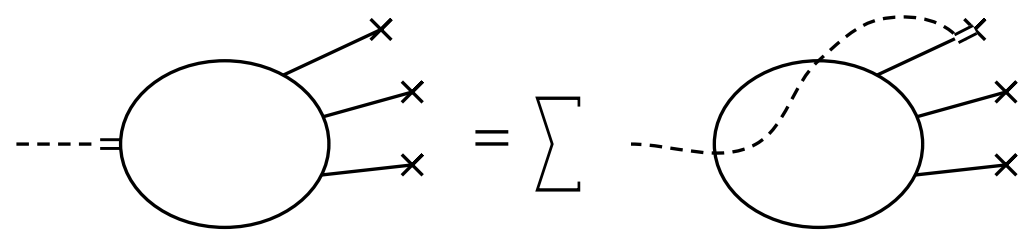

The summation is over the various sources to which the dotted line can be attatched.

The derivation went the same way as that for my own identities. The only reason why I had not written these identities in this new form before was that I thought the extra pieces would be cumbersome, requiring new renormalization counterterms of their own, and, furthermore, I didn't need them. I think I was still suffering from the indoctrination that infinities should be avoided at all costs. It is clear now that these newer identities are more complete. And so it happened that they were to become known as the Slavnov-Taylor identities, a pivotal property of any gauge theory.

\section{The Higgs-Kibble Mechanism and Dimensional Renormalization.}

For my advisor, Veltman, all this was just Spielerei. Massless Yang-Mills fields seem not to occur in Nature. They are just there for exercises. The real thing is the massive case, and he thought that that would be an entirely different piece of cake. Actually however, the step remaining to be taken was a small one [32]. As I knew from Cargèse, the actual nature of the vacuum state has little effect upon renormalization counter terms. All needed to be done is to add to the gauge invariant Lagrangian the by now familiar Higgs terms,

$$
\mathcal{L}^{\text {Higgs }}=-\frac{1}{2}\left(D_{\mu} \phi\right)^{2}-V(\phi)
$$

where $V(\phi)$ has the familiar dumb-bell shape, just like in the Gell-Mann Lévy sigma model (for simplicity I take the $\phi$ field here to be a real multiplet). Writing $\phi=F+\eta$ we get a gauge invariant Lagrangian for the $B$ and $\eta$ fields, such that now the $B$ fields get the required mass. To appease Veltman I wrote the self-interaction as

$$
V=\frac{1}{8} \lambda\left(2 F \eta+\eta^{2}\right)^{2}
$$

so that at least at lowest order the vacuum energy density vanishes. In terms of the $\eta$ 
fields the gauge transformation laws for these and the $B$ field look very similar:

$$
\begin{aligned}
B_{\mu}^{a \prime} & =B_{\mu}^{a}+f^{a b c} \Lambda^{b} B_{\mu}^{c}-\frac{1}{g} \partial_{\mu} \Lambda^{a} ; \\
\eta^{\prime} & =\eta+T^{a} \Lambda^{a} \eta+T^{a} \Lambda^{a} F .
\end{aligned}
$$

Everything else went exactly as in the previous paper. Because the local gauge invariance is still exact we again have Slavnov-Taylor identities, BRS invariance and from them one can prove unitarity and equivalence of the various gauge choices. A judicious gauge choice was found such that the propagators for the massive gauge fields and the other fields became as simple as possible.

The problem of regularizing and renormalizing diagrams with two or more loops was still there. Veltman and I discussed a lot about this problem and eventually agreed what the best strategy was: continuous variation of the number of space-time dimensions [33]. Upon Veltman's explicit instructions, both verbally and in writing [34], I refrain from commenting about who of us did what in that paper (although one doesn't forget that easily, as he claims).

As if it were a seed from outer space, the idea of making the dimensionality of spacetime continuous germed simultaneously in various places as an answer to different problems. Kenneth G. Wilson and Michael E. Fischer [35] were writing a paper proposing to calculate critical phenomena in statistical physics in $4-\varepsilon$ dimensions as an expansion in $\varepsilon$. And independently of us C. Bollini and J. Giambiagi [36], and J. Ashmore [37], also suggested to use analyticity in space-time dimensions as a regulator.

Dimensional regularization does not work if there is an Adler-Bell-Jackiw anomaly. Initially I thought that this could not be more than a technical difficulty, but it was quickly pointed out that indeed if these anomalies do not cancel gauge theories cannot be renormalized at all [38]; they are sick. Consequently in all gauge theories one must impose the new and important requirement that these chiral anomalies cancel out against each other. Only at later times the physical reasons for this requirement became evident: it has everything to do with the appearance of instantons in the theory. The symmetries destroyed by them cannot be gauged.

It may be noticed that by now I entirely address the problem of renormalization as a procedure for infinity subtraction. As explained in the beginning this is not at all what renormalization really is from a physical point of view. It is preferable to talk about regularization first, and then renormalization afterwards. Regularization is the replacement of a theory by a slightly mutilated model, using a cut-off. In such a model the observed parameters are finite and related in some calculable way to the "bare" parameters. 
We must show that, even if the relations between the observed and the bare parameters tend to become divergent, the effects of the cut-off become negligible at large distance scales. One only needs to demonstrate that in terms of the observed, "renormalized" observables the limit where the cut-off goes away exists and is perturbatively finite ${ }^{\dagger}$. It does not matter much how crazy the mutilation was in the beginning, as long as the limit is well-behaved. Going to $4-\varepsilon$ dimensions is just such a crazy regularization scheme. It turns out to be technically extremely elegant. Anyway, the important thing was that this method works fine at all orders of perturbation expansion and not just up to one loop, like the 5 dimensional procedure found earlier.

We now had a general scheme for producing theories with interacting massive vector particles . At first I was thinking about applying it to rho mesons, as a nice generalization of the Gell-Mann Lévy sigma model. But of course Veltman could convince me that the weak interactions were a much more promising application. I had essentially reproduced Weinberg's model before I saw his 1967 paper [39]. Veltman brought it with him when he returned from Geneva. Anyway, when my paper on the Massive Yang-Mills fields came out I soon received letters from both Weinberg and Salam [40] with copies of their 1967 papers, in which the renormalizability of weak interaction theories of this nature had been speculated on.

More important to my mind was that we now had a large class of renormalizable models with massive and massless vector mesons. A crucial argument was added to this by Chris Llewellyn Smith [41] and J. Cornwall, D. Levin and George Tiktopoulos [42] : they showed that requiring unitarity implies that the only such models are gauge theories. So not only do we have a large class of new models, we have the complete class of renormalizable vector theories.

\section{The Renormalizability Requirement.}

Having been so successful in formulating the renormalizability requirement and introducing this in realistic theories for the weak interaction it would have been natural to promote renormalizability to the status of a primary principle in quantum field theory. Requiring such a theory to be renormalizable indeed limits us to a subclass large enough to enable us to include all particle and interaction types known, and small enough to be fantastically predictive. The extremely accurate tests of the Standard Model at facilities such as LEP confirm the basic correctness of such a standpoint.

\footnotetext{
$\dagger$ non-perturbatively the limit might not exist. This happens for instance in $\lambda \phi^{4}$ theory. Such a theory is perturbatively fine but non-perturbatively non-consistent. Curiously, such models are still extremely useful in physics; their predictions are accurate but cannot be infinitely accurate, see sect. 6 .
} 
Nevertheless such a dogmatic attitude would obscure some important aspects of quantum field theories in general. It cannot be ignored that even renormalized theories suffer from weaknesses undermining their status as ultimate descriptions of reality. What I am referring to here is the fact that these theories can be rigorously formulated as perturbative expansions in terms of small coupling parameters, but most of them will probably not allow any meaningful definition at all beyond this perturbative level. There is little harm in this in practice because the margins within which these theories are ill defined will be of order $e^{-C / g^{2}}$ where $g$ is the coupling constant and $C$ some characteristic number related to the theory's $\beta$-functions.

It is safe to say that at present there exists no mathematically 'perfect' quantum field theory at all in four space-time dimensions (unless it were to describe only free particles). At least five degrees of sophistication can be distinguished:

1. Non-renormalizable theories. These are theories for which the amplitudes can only be computed at tree level:

$$
\Gamma=a_{1} g^{2}+\mathcal{O}\left(g^{4}\right)
$$

The higher order quantum corrections of order $g^{4}$ would require new and unknown subtraction constants. Examples are: the old Fermi weak interaction theory in hich four fermions are coupled at single points, and gravitation coupled to matter. Note that even these theories, in spite of their limitations, have proven their worths as stages of understanding in physics. But the region of applicability ends at energy scales of order of $1 / g$ times the typical mass scale.

2. One-loop renormalizable theories:

$$
\Gamma=a_{1} g^{2}+a_{2} g^{4}+\mathcal{O}\left(g^{6}\right)
$$

No new counter terms are needed even at the one loop level. Examples are pure gravity and massive Yang-Mills fields with the Higgs particle omitted.

3. Renormalizable theories. No new counter terms at any finite order. Examples are QED, $\lambda \phi^{4}$ theory and all gauge theories. The asymptotic expansion is well defined:

$$
\Gamma=\sum_{n} a_{n} g^{2 n}+\mathcal{O}\left(e^{-C / g^{2}}\right)
$$

but the marginal non-perturbative correction terms are still beyond control.

4. Asymptotically free theories [43]. In these theories all running coupling constants tend to zero as the energies go to infinity. In these theories the renormalization counter terms can be rigorously defined in the limit of a vanishing cut-off, and in view of this 
one may well suspect that these theories can be definied rigorously mathematically. Unfortunately there is no proof of that ${ }^{\dagger}$. Indeed, such theories are usually not Borel resummable. Examples are: QCD and some special renormalizable models containing not only gauge fields but also scalars and spinors.

5. Borel resummable theories. In these theories one redefines the amplitudes $\Gamma$ as

$$
\Gamma=\int_{0}^{\infty} \mathrm{d} z e^{-z / g^{2}} B(z),
$$

where $B(z)$ has a convergent expansion in powers of $z$, but it has to be proven that it is well-defined for all $z$. There are no known non-trivial examples in four dimensions, but an asymptotically free model in the planar limit $(N \rightarrow \infty)$ behaves this way [54].

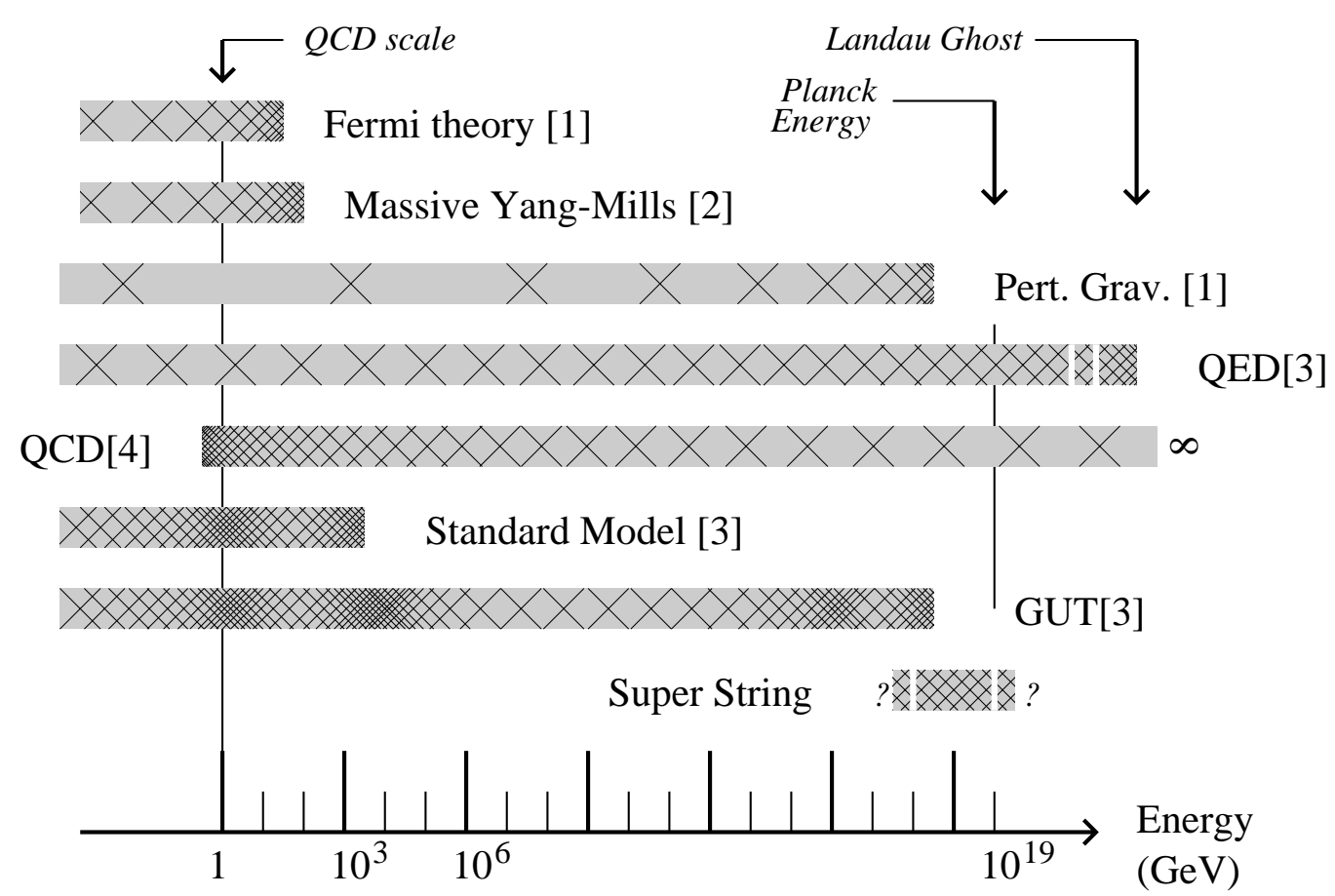

Fig. 6. Comparison of the domains of validity for different quantum field theories. Shading indicates amount of structure in a model. Numbers correspond to the position in the list of increasing sophistication.

$\dagger$ The difficulty one encounters when trying to prove mathematical completeness of an asymptotically free theory reside in the a priori unknown characteristics of the vacuum state 
A theory is more sophisticated if it allows for more structure in a larger domain of different energy scales. If the structure becomes too complex the mathematical rigour breaks down because of lack of convergence of the known expansion techniques for their analysis. A theory further down the list I just gave allows for more structure along a larger domain of energies. See Fig. 6 in which the coloration indicates structure.

In summary: the more complex the particle spectrum and interactions are, and the more rigid our demands for precision, the stronger the constraints on a theory. Renormalizability is not sufficient to make a theory completely airtight but it provides us with a paradigm that allows consistent calculations with margins that decrease exponentially as the interaction strengths decrease. All our theories have a built-in twilight zone, where, according to their own logic, the calculational rules fail. In these regions our theories will have to be supplanted by others, as has always been the case in physics. It is suspected, but has never been proven, that asymptotically free theories have no such twilight zone. If one merely has a renormalizable theory, the twilight zone is exponentially far away.

\section{Further developments}

In practice this new development was an enormous improvement. Within just a couple of years many further insights were obtained. A charming feature of many versions of 'unified gauge theories' is that they allow for entirely new kinds of solutions for their field equations corresponding to particles with a single magnetic (north or south) charge: magnetic monopoles [44]. Independently of the present author the discovery was made when Alexander A. Polyakov introduced his "hedgehog solutions" [45]. As he states in a footnote of his paper, the fact that his solution possesses magnetic charge was pointed out to him by Lev Okun'.

Further search brought to light that there exist also four-dimensional localized field solutions [46] in all non-Abelian gauge theories. When I introduced the name 'instantons' for these the word was censored by Phys. Rev. Letters [47]. They wanted to change it in: "Euclidean Gauge Pseudoparticle Solution, EGPS for short". When I protested they went as far as "Euclidean Gauge Soliton (EGS)". These ugly acronyms only hastened the general acceptance of 'instanton' ever since. My interest in the instanton, and its peculiar effects on fermions, came from the fact that these effects are exponential in $-1 / g^{2}$. How can this be squared with the observation of the previous chapter, which was that effects this small need not be calculable at all in a renormalizable field theory? I decided to go through a complete calculation to check whether there would be any residual infinities or other ambiguities [48]. There were none. Just because instanton effects either violate a symmetry such as baryon number conservation or lift a degeneracy such as in pure QCD, 
the effects turn out to be totally unambiguous.

It is a characteristic of successful theories that they provide further understanding in many different areas of the field, in elegant and unsuspected ways. As for the Standard Model, we now know that the roles of asymptotic freedom, monopoles and instantons are crucial in our present picture of quark confinement, the hadron spectrum, the scaling phenomena and jet physics. The renormalized theory allows us to reproduce the observed data on the $\mathrm{Z}$ and $\mathrm{W}$ bosons with unprecedented precision. The Standard Model, as a gauge theory with fermions and at most only one scalar, is indeed tremendously successful. My presentation was sketchy. Subjects that, among others, should have been discussed as well or in more detail, but for which I had no time left, are:

- The discovery of asymptotic freedom [43, 49];

- Renormalization of quantum gravity [50];

- Quark confinement [51];

- Constructive Field Theory [52];

- Appelquist-Carrazone decoupling [53];

- The $N \rightarrow \infty$ limit and the resulting planar diagram approach to QCD [54];

- Naturalness and the instanton index matching condition [55];

- $B+L$ violation [56] and monopole catalysis [57];

- Renormalons [58].

All these topics are closely linked to the renormalization issue. Of course, after two decades have past, also the deficiencies in our theories are standing out clearly: a theory that should explain why the local symmetry is as it is, where the fermion spectrum comes from and how the values of about 20 constants of nature are determined, is still being searched for, but it is difficult to believe that a giant leap in particle theory, as it occurred in the 70's, will be repeated in the near future.

\section{References}

1. G. 't Hooft, "Renormalization of Gauge Theories", in Proceedings of the Third International Symposium on the History of Particle Physics: 'The Rise of the Standard Model", SLAC, June 24-27, 1992.

2. H.A. Bethe, Phys. Rev. 72 (1947) 339.

3. J. Schwinger, Phys. Rev. 73 (1948) 416, ibid. 74 (1948) 1439.

4. S. Tomonaga, Progr. Theor. Phys. 1 (1946) 27.

5. R.P.Feynman, Rev. mod. Phys. bf 20 (1948) 367; id, Phys. Rev. 74 (1948) 939, 1430.

6. F.J. Dyson, Phys. Rev. 85 (1952) 631. 
7. R.L. Mills and C.N. Yang, Suppl. Progr. Theor. Phys. 37 and 38 (1966) 507.

8. H. Kramers, Quanten theorie des Elektrons und der Strahlung, Akad.Verlag, Leipzig (transl. D. ter Haar, North Holland, Amsterdam, 1957); id, A review talk at the Shelter Island conference (June 1947), unpubl. See S.S. Schweber, "A short history of Shelter Island I", in Shelter Island II (eds. R. Jackiw, N.N. Khuri, S. Weinberg and E. Witten, MIT Press, Cambridge, Mass, 1985). See also his Collected scientific papers (Amsterdam 1956) 333 and 347.

9. R. de L. Kronig, Journ. Opt. Soc. Amer. 12 (1926) 547.

10. N.G. van Kampen, Ned. T. Natuurk. 24, Januari 1958 and Februari 1958 (in Dutch).

11. T.Y. Cao and S.S. Schweber, "The Conceptual Foundation and Philosophical Aspects of Renormalization Theory", Brandeis and Harvard Universities preprint.

12. M. Veltman, Physica 29 (1963) 186; G. 't Hooft and M. Veltman, "DIAGRAMMAR", CERN Report 73/9 (1973), reprinted in "Particle Interactions at Very High Energies", Nato Adv. Study Inst. Series, Sect. B, vol. 4b, p. 177; G. 't Hooft, "Gauge Field Theory", in Proceedings of the Adriatic Meeting, Rovinj 1973, ed. M. Martinis et al, North Holland / Am. Elsevier, p.321; G. 't Hooft, "DIAGRAMMAR and Dimensional Renormalization, in "Renormalization and Invariance in Quantum Field Theory", Capri Summer Meeting July 1973, ed. E.R. Caianiello, Plenum New York 1974, p. 247.

13. O. Klein, in "New Theories in Physics", Conference organised in collaboration with the International Union of Physics and the Polish Intellectual Co-operation Committee, Warsaw, May 30th - June 3rd, 1938.

14. C.N. Yang and R.L. Mills, Phys. Rev. 96 (1954) 191, see also R. Shaw, Cambridge Ph.D. Thesis, unpublished.

15. C.N. Yang, Selected Papers 1945-1980 With Commentary, Freeman and Co., San Francisco 1983, p. 20 .

16. R.P. Feynman, Acta Phys. Polonica 24 (1963) 697.

17. S.L. Glashow, Nucl. Phys. 22 (1961) 579.

18. M. Veltman, Nucl. Phys. B7 (1968) 637; J. Reiff and M. Veltman, Nucl. Phys. B13 (1969) 545; M. Veltman, Nucl. Phys. B21 (1970) 288.

19. Proceedings of the Cargèse Lectures in Physics, Vol. 5, Gordon and Breach, New York, London, Paris, 1972, ed. D.Bessis.

20. M. Gell-Mann and M. Lévy, Nuovo Cim. 16 (1960) 705.

21. B.W. Lee, Nucl. Phys. B9 (1969) 649; B.W. Lee, "Chiral Dynamics", Gordon and Breach, New York, London, Paris 1972.

22. J.-L. Gervais and B.W. Lee, Nucl. Phys. B12 (1969) 627.

23. K. Symanzik, Let. Nuovo Cim. 2 (1969) 10, id, Commun. Math. Phys. 16 (1970) 48.

24. L.D. Faddeev and V.N. Popov, Phys. Lett. 25B (1967) 29. See also: L.D. Faddeev, Theor. and Math. Phys. 1 (1969) 3 (in Russian) L.D. Faddeev, Theor. and Math. Phys. 1 (1969) 1 (Engl. 
transl).

25. S. Mandelstam, Phys. Rev. 175 (1968) 1580, 1604.

26. G. 't Hooft, Nucl. Phys. B33 (1971) 173.

27. C. Becchi, A. Rouet and R. Stora, Commun. Math. Phys. 42 (1975) 127; id., Ann. Phys.(N.Y.) 98 (1976) 287. See also: I.V. Tyutin, Lebedev Prepr. FIAN39 (1975), unpubl.; R. Stora, Cargèse lectures 1976; J.Thieri-Mieg, J.Math.Phys. 21 (1980) 2834; L. Beaulieu, and J. Thieri-Mieg, Nucl. Phys. B197 (1982) 477.

28. S.L. Adler, Phys. Rev. 177 (1969) 2426; see also H. Fukuda and Y. Miyamoto, Progr. Theor. Phys. 4 (1949) 347.

29. J.S. Bell and R. Jackiw, Nuovo Cim. A60 (1969) 47.

30. A. Slavnov, Theor. Math. Phys. 10 (1972) 153 (in Russian), Theor. Math. Phys. 10 (1972) 99 (Engl. Transl.)

31. J.C. Taylor, Nucl. Phys. B33 (1971) 436.

32. G. 't Hooft, Nucl. Phys. B35 (1971) 167.

33. G. 't Hooft and M. Veltman, Nucl. Phys. B44 (1972) 189.

34. M. Veltman, invited talk given at the Third International Symposium on the History of Particle Physics, SLAC, June 24-27, 1992, section 15.

35. K.G. Wilson, Phys. Rev. D3 (1971) 1818; K.G. Wilson and M.E. Fisher, Phys. Rev. Lett. 28 (1972) 240.

36. C. Bollini and J. Giambiagi, Nuovo Cim. 12B (1972) 20.

37. J. Ashmore, Nuovo Cim. Lett 4 (1972) 289.

38. S.L. Adler and W.A. Bardeen, Phys. Rev. 182 (1969) 1517; W.A. Bardeen, Phys. Rev. 184 (1969) 1848; D.G. Boulware, Phys. Rev. D11 (1975) 1404; Phys. Rev. D13 (1976) 2169.

39. S. Weinberg, Phys. Rev. Let. 19 (1967) 1264.

40. A. Salam and J.C. Ward, Phys. Lett. 13 (1964) 168; A. Salam, in: Elementary Particle Theory, ed. N. Svartholm (Stockholm, 1968).

41. Ch. Llewellyn-Smith, Phys. Lett. B46 (1973) 233.

42. J. Cornwall, D. Levin and G. Tiktopoulos, Phys. Rev. Lett. 30 (1973) 1268.

43. G. 't Hooft, announcement made at the Colloquium on Renormalization of Yang-Mills Fields, C.N.R.S., Marseille, June 19-23, 1972; D.J. Gross and F. Wilczek, Phys. Rev. Lett. 30 (1973) 1343; H.D. Politzer, Phys. Rev. Lett. 30 (1973) 1346; G. 't Hooft, Nucl. Phys. B61 (1973) 455; H.D. Politzer, Phys. Rep. 14c (1974) 129.

44. G. 't Hooft, Nucl. Phys. B79 (1974) 276; Nucl. Phys. B105 (1976) 538.

45. A.M. Polyakov, JETP Lett. 20 (1974) 194.

46. A.A. Belavin, A.M. Polyakov, A.S. Schwartz and Yu.S. Tyupkin, Phys. Lett. 59B (1975) 85. 
47. G. 't Hooft, Phys. Rev. Lett. 37 (1976) 8; 3432; R. Jackiw and C. Rebbi, Phys. Rev. Lett. 37 (1976) 172; C.G. Callan Jr., R.F. Dashen and D.J. Gross, Phys. Lett. 63B (1976) 334; Phys. Rev. D17 (1978) 2717.

48. G. 't Hooft, Phys. Rev. D14 (1976) 3432; Err. Phys. Rev. D18 (1978) 2199.

49. K. Symanzik, Nuovo Cim. Lett. 6 (1973) 77 K. Symanzik, Commun. Math. Phys. 18 (1970) 227 K. Symanzik, Commun. Math. Phys. 23 (1971) 49 K.G. Wilson, Phys. Rev.D3 (1971) 1818 G. 't Hooft, Nucl. Phys.B62 (1973) 444; Nucl. Phys. B254 (1985) 11; Phys. Lett.109B (1982) 474; ibid. 119B (1982) 369 .

50. G. 't Hooft and M. Veltman, Ann. Inst. Henri Poincaré, 20 (1974) 69.

51. S. Mandelstam, Phys. Rep. 23 (1976) 237; H.J. Lipkin, Phys. Lett. 45B (1973) 267; M. Fritsch, M. Gell-Mann and H. Leutwyler, Phys. Lett. 47B (1973) 365; C.G. Callan, R. Dashen and D. Gross, Phys. Lett. 66B (1977) 375; C.G. Callan, R. Dashen and D. Gross, Phys. Lett. 78B (1978) 307; A.M. Polyakov, Nucl. Phys. B120 (1977) 429; G. 't Hooft, Phys. Scripta 24 (1981) 841; Nucl. Phys. B190 (1981) 455.

52. K. Osterwalder and R. Schrader, Commun. Math. Phys. 31 (1973) 83; ibid. 42 (1975) 281.

53. T. Appelquist and J. Carazzone, Phys. Rev. D11 (1975) 2856.

54. G. 't Hooft, Nucl. Phys. B72 (1974) 461; G. 't Hooft, Nucl. Phys. B75 (1974) 461 Commun. Math. Phys. 86 (1982) 449; ibid. 88 (1983) 1.

55. G. 't Hooft, in "Recent Developments in Gauge Theories", Cargèse 1979, ed. G. 't Hooft et al., Plenum Press, New York, 1980, Lecture III, reprinted in: "Dynamical Symmetry Breaking, a Collection of reprints", ed. A. Fahri et al., World Scientific, Singapore, Cambridge, 1982, p. 345.

56. A. Ringwald, Nucl. Phys. B330 (1990) 1; L. McLerran, A. Vainshtein and M. Voloshin, Phys. Rev. D42 (1990) 171.

57. V. Rubakov, JETP Lett 33 (1981) 644; Nucl. Phys. B 203 (1982) 311; C.G. Callan, Phys. Rev. D25 (1982) 2141, D26 (1982) 2058; Nucl. Phys. B212 (1983) 391.

58. G. 't Hooft, in "The Whys of Subnuclear Physics", ed. A. Zichichi, Plenum, New York/London, p. 943. 